\title{
Functionalized magnetic biopolymeric graphene oxide with outstanding performance in water purification
}

\author{
Amit Kumar Sarkar $\mathbb{B}^{1}$, John Kwame Bediako ${ }^{1}$, Jong-Won Choi ${ }^{1}$ and Yeoung-Sang Yun (D)
}

\begin{abstract}
Graphene oxide (GO) is an attractive material for water treatment, although its high surface energy restricts its practical application. To overcome this challenge, we have developed a well-dispersed and interconnected, functionalized biopolymeric $\mathrm{GO}$ with magnetic activity, namely, $\mathrm{cl}-\mathrm{CS}-\mathrm{p}(\mathrm{MA}) / \mathrm{Fe}_{3} \mathrm{O}_{4} \mathrm{NPs}$. This three-dimensional, sponge-like nanostructured material is composed of graphene oxide nanosheets dispersed in functionalized chitosan/poly (methacrylic acid) and is cross-linked with in situ-developed $\mathrm{Fe}_{3} \mathrm{O}_{4}$ nanoparticles. Methacrylic acid (MA)-functionalized chitosan (CS) cross-linked with N,N-methylenebis(acrylamide) (MBA), designated as $\mathrm{Cl}-\mathrm{CS}-\mathrm{p}(\mathrm{MA})$, facilitates the stable dispersion of $\mathrm{GO}$ nanosheets, providing a proper solid matrix for the generation of well-dispersed in situ $\mathrm{Fe}_{3} \mathrm{O}_{4} \mathrm{NPs}_{\mathrm{s}}$. The methodology allows for the generation of numerous binding sites with an interconnected morphology, facilitating the rapid uptake of a cationic dye in significant quantity, e.g., methylene blue (MB), which is used as a model water pollutant. In this work, the structural architecture of $\mathrm{cl}-\mathrm{CS}-\mathrm{p}(\mathrm{MA}) / \mathrm{Fe}_{3} \mathrm{O}_{4} \mathrm{NPs}$ was characterized with multiple approaches, and the adsorption mechanism was revealed to be an electrostatic interaction. The synthesized nanocomposite showed significant recyclability and structural stability. Adsorption equilibrium was achieved within $20 \mathrm{~min}$, and the maximum adsorption capacity was $2478 \mathrm{mg} / \mathrm{g}$ for MB, surpassing the values reported for any other adsorbents to date.
\end{abstract}

\section{Introduction}

The rapid development of nanotechnology and the design of nanostructured materials have led to noteworthy breakthroughs in various fields and applications ${ }^{1,2}$. In macroscopic structures, the effective growth of nanoparticles (NPs) introduces synergy in novel assemblies of nanostructured composites, which can be used to translate unique properties from individual NPs to nanostructured materials ${ }^{3}$. Such nanostructured materials are enriched with tunable surface functionalities, structural stability, and high adsorption capacities ${ }^{4}$. By taking advantage of these features, the performance of wastewater remediation technology could be significantly enhanced by using nanostructured materials. The efficient

\footnotetext{
Correspondence: Yeoung-Sang Yun (ysyun@jbnu.ac.kr)

${ }^{1}$ School of Chemical Engineering, Chonbuk National University, Jeonju

561-756, Republic of Korea
}

treatment of wastewater has the potential to minimize the scarcity of clean water in domestic, agricultural, and industrial perspectives.

Among the various types of water pollutants, colored organic dyes pose significant threats to human health and the environment and are aesthetically objectionable ${ }^{5}$. The discharge of untreated dye wastewater, even at a very low concentration $(<1 \mathrm{mg} / \mathrm{L})$, is unacceptable. Among the various technologies that have been deployed for dye removal, adsorption is considered to be one of the easiest, most effective, and inexpensive methods ${ }^{6}$. An efficient adsorbent must have sufficient chemical stability, have enough active adsorption sites for effective contact with adsorbates, and be recyclable to enable easy separation and regeneration of the adsorbent under experimental conditions. The conventionally activated carbons and natural zeolites have been used as adsorbent materials in 
past studies ${ }^{7,8}$. However, such materials show low surface functionalities, limited adsorption capacities, and slow kinetics. Therefore, the fabrication of a single adsorbent material with a high adsorption capacity, rapid kinetics, structural stability, and recyclability remains a strategic challenge.

Nanostructured composite materials have attracted significant attention as new-generation adsorbents for dye removal. Examples include clays, exfoliated titanate layers, mesoporous carbons, carbon nanofibers, and polyanilines, all of which have been used as the main constituent of nanocomposite/composite building blocks ${ }^{9-14}$. Among these materials, graphene oxide (GO), a two-dimensional (2D) carbon nanomaterial, offers attractive and unique physicochemical properties ${ }^{15}$ and is considered an excellent adsorbent ${ }^{16}$. However, the use of GO as an adsorbent has been restricted because of the high surface energy of GO sheets, which can lead to aggregation through van der Waals interactions, in addition to the ultrahigh centrifugation needed to collect GO after pollutant removal ${ }^{17}$. Consequently, modified GOs have been used in aqueous media to increase the separation efficiency of GO-based adsorbents; examples include threedimensional GO sponges, a magnetic GO/poly(vinyl alcohol) composite gel, and poly(acrylic acid)-modified magnetic GO with reported MB dye adsorption capacities of 467, 270.94, and $290.7 \mathrm{mg} / \mathrm{g}$, respectively ${ }^{17-19}$. Although the reported GO-based adsorbents may eventually overcome the separation problem, they lack the significant uptake capacity necessary for handling aqueous pollutants. The subsequent assembly of GO into macroscopic structures yielded a better adsorption performance; for example, nanoporous GO sponges and GO beads were effective for the removal of toxic organic solvents and hazardous chemicals from water ${ }^{20,21}$. Polymeric brushes of poly(acrylamide) grafted onto reduced GO sheets behaved as an effective adsorbent for $\mathrm{MB}$ $(1530 \mathrm{mg} / \mathrm{g})$ and $\mathrm{Pb}^{2+}(1000 \mathrm{mg} / \mathrm{g})^{22}$. Recently, GO/chitosan (CS) microspheres were developed for the rapid removal of $\mathrm{MB}(584.6 \mathrm{mg} / \mathrm{g})$ and $\mathrm{Cr}(\mathrm{VI})(292.8 \mathrm{mg} / \mathrm{g})^{23}$.

Numerous efforts have been made to develop functional and recyclable GO-based adsorbents. However, an adsorbent that meets the required specifications, such as rapid achievement of equilibrium and excellent adsorption capacity, separation ability, and recyclability, has not yet been developed. Bearing in mind the aforementioned challenges for a high-performance advanced adsorbent, we developed a novel synthesis strategy to obtain crosslinked polymeric hybrid nanocomposites, namely, $\mathrm{cl}-\mathrm{CS}-\mathrm{p}(\mathrm{MA}) / \mathrm{Fe}_{3} \mathrm{O}_{4} \mathrm{NPs}$. The nanocomposite was prepared by unstacking GO nanosheets by infiltrating with methacrylic acid (MA)-functionalized chitosan (CS) cross-linked with $\mathrm{N}, \mathrm{N}^{\prime}$-methylenebis(acrylamide) (MBA), designated as $\mathrm{cl}-\mathrm{CS}-\mathrm{p}(\mathrm{MA})$, and cross-linking with in situ-generated $\mathrm{Fe}_{3} \mathrm{O}_{4} \mathrm{NPs}$. CS, a cationic biopolymer of glucosamine and $\mathrm{N}$-acetylglucosamine, shows $\mathrm{pH}$ triggered self-assembly responsiveness ${ }^{24}$. Herein, the implemented approach is discussed for the first time. To understand the individual role of each component in the nanocomposite, controlled adsorption studies were carried out using cl-CS-p(MA), cl-CS-p(MA)/GO (without $\mathrm{Fe}_{3} \mathrm{O}_{4}$ ), and cl-CS-p(MA)/ $/ \mathrm{Fe}_{3} \mathrm{O}_{4}$ (without GO). We are delighted to report that the synthesized cl-CS-p(MA)/ $\mathrm{GO}-\mathrm{Fe}_{3} \mathrm{O}_{4} \mathrm{NPs}$ can rapidly capture the cationic MB dye and showed an outstanding $\mathrm{MB}$ dye adsorption capacity of $2478 \mathrm{mg} / \mathrm{g}$, which is the highest reported value to date. The excellent adsorptive characteristics of this nanocomposite arise from the combined synergistic effect of well-dispersed active binding sites and interconnected microchannels, which facilitate rapid adsorption. More interestingly, the developed adsorbent was structurally stable over the entire $\mathrm{pH}$ range (pH 3-9) and can easily be separated using an external magnet and spatula.

\section{Materials and methods \\ Materials}

Chitosan (CS) (medium molecular weight; product of USA), potassium persulfate (KPS) (product of Germany), methacrylic acid (MA) (product of USA), graphite (powder, $<20 \mu \mathrm{m}$ ), iron (III) chloride hexahydrate (product of China), iron (II) sulfate heptahydrate (product of USA), and methylene blue (MB) $\left(\mathrm{C}_{16} \mathrm{H}_{18} \mathrm{ClN}_{3} \mathrm{~S} .3 \mathrm{H}_{2} \mathrm{O} ; \mathrm{FW}\right.$ 373.9; product of USA) were procured from Sigma Aldrich. N,N'-methylenebisacrylamide (MBA), potassium permanganate, acetone, ammonia, sulfuric acid, sodium hydroxide (Samchun Pure Chemicals, Korea), acetic acid (Junsei Chemicals Co. Ltd., Japan), methyl orange (MO) $\left(\mathrm{C}_{14} \mathrm{H}_{14} \mathrm{~N}_{3} \mathrm{NaO}_{3} \mathrm{~S}\right)$ (Showa Chemicals, Japan), methanol (Honeywell Burdick \& Jackson, Korea), and toluidine blue (TB) (Sigma Aldrich, India) were of analytical grade and used as received. Deionized [Millipore/direct-Q3UV (Millipore, USA)] water was used throughout the experiment.

\section{Preparation of cross-linked chitosan [cl-CS-p(MA)]}

The cross-linked chitosan was synthesized using a free radical polymerization approach. Chitosan $(0.5 \mathrm{~g})$ was dissolved in $80 \mathrm{~mL}$ of $0.1 \mathrm{M}$ acetic acid solution in a threenecked round-bottom (RB) flask. The RB flask was positioned in an electrically operated oil bath, and a thermometer was used to maintain the temperature at $65^{\circ} \mathrm{C}$ throughout the reaction. Then, the whole system was placed over a magnetic stirrer for continuous stirring at $350 \mathrm{rpm}$. After maintaining an inert atmosphere of $\mathrm{N}_{2}$ in the RB flask, $3.699 \times 10^{-5}$ mol of KPS was added. Successively, after $10 \mathrm{~min}$ of intervals, $0.1178 \mathrm{~mol}$ of MA followed by $3.243 \times 10^{-5} \mathrm{~mol}$ of MBA were carefully added into the reaction system. The cross-linking 
copolymerization reaction was allowed to continue up to $3 \mathrm{~h}$ of reaction time, maintaining the same reaction temperature while stirring. Once the reaction mixture had turned to a thick white gel, the polymerization was terminated using an aqueous solution of saturated hydroquinone. Subsequently, the cooled white gel was washed with $400 \mathrm{~mL}$ of 1:1 methanol-acetone mixture to remove the unreacted monomer and homo polymer. Finally, the washed product was dried using a freeze dryer.

\section{Synthesis of $\mathrm{cl}-\mathrm{CS}-\mathrm{p}(\mathrm{MA}) / \mathrm{GO}-\mathrm{Fe}_{3} \mathrm{O}_{4} \mathrm{NPs}$}

GO was prepared using a modification of Hummer's method (experimental details in the Supporting Information $)^{25,26}$. A gelling matrix of cl-CS-p(MA) $(1 \mathrm{~g})$ was prepared in $100 \mathrm{~mL}$ of $0.01(\mathrm{~N}) \mathrm{NaOH}$ solution at $65^{\circ} \mathrm{C}$ with constant stirring. Next, a fixed quantity of GO $(0.06 \mathrm{~g})$ in $10 \mathrm{~mL}$ of water was placed under sonication for $15 \mathrm{~min}$. Afterward, the aqueous solution of GO was added into the gelling matrix using a dropping funnel under the presence of $\mathrm{N}_{2}$ atmosphere at $65^{\circ} \mathrm{C}$ for $15 \mathrm{~h}$. Next, $5 \mathrm{~mL}$ of each aqueous solution of $0.278 \mathrm{~g}$ of ferrous sulfate $\left(\mathrm{FeSO}_{4}\right)$ and $0.540 \mathrm{~g}$ of ferric chloride $\left(\mathrm{FeCl}_{3}\right)$ was added while maintaining the same experimental conditions, followed by the addition of $9 \mathrm{~mL}$ of ammonia solution. After $6 \mathrm{~h}$ of continuous stirring, the reaction mixture was allowed to cool and then was precipitated in acetone, resulting in a black spongy material. The material was washed with water and placed in a desiccator overnight at room temperature.

\section{Characterizations}

Fourier transform-infrared spectrometry (FT-IR) (Jasco FT/IR-4100, Japan) was performed using a scan range of $400-4000 \mathrm{~cm}^{-1}$ with pressed $\mathrm{KBr}$ pellets. Powder X-ray diffraction (XRD) spectra were collected with a scan range of $5-80^{\circ}$ and scan rate of $2^{\circ} / \mathrm{min}$ (X'pert PRO, PANalytical, Netherlands), installed in the Center for UniversityWide Research Facility (CURF), Chonbuk National University, Korea. Surface morphologies were imaged using field-emission scanning electron microscopy (FE-SEM) coupled with energy-dispersive spectroscopy (EDS) (SUPRA 40VP, Carl Zeiss, Germany) and a CS-corrected field-emission transmission electron microscope (JEOL, JEM-ARM-200F, Japan) from CURF. X-ray photoelectron spectroscopy (XPS) was carried out using K-Alpha spectrometer (ThermoFisher Scientific, UK) with monochromated $\mathrm{Al} \mathrm{K} \alpha$ radiation $(1486.6 \mathrm{eV})$ and peaks were deconvoluted/optimized using XPSPEAK 4.1 software. Raman spectroscopy was performed using a Nanofinder 30 (Tokyo Instrument, Japan), from Korea Basic Science Institute (KBSI), Jeonju center, Korea. The point of zero charge $\left(\mathrm{pH}_{\mathrm{pzc}}\right)$ of cl-CS-p(MA)/GO-Fe $\mathrm{O}_{4} \mathrm{NPs}$ was measured using the $\mathrm{pH}$ drift method ${ }^{27}$ (the procedure is described in the Supporting Information). The specific surface area and mean pore size were measured by the Brunauer-Emmett-Teller (BET) method (BELSORPmax/MicrotracBEL Corp., Japan at $77 \mathrm{~K}$ ). The magnetic property of the synthesized nanocomposite was recorded using a vibrating sample magnetometer (VSM, Lakeshore 7404, USA) operated at room temperature. Adsorption studies were done using UV-VIS spectrophotometer (UV2550, Shimadzu, Japan).

\section{Dye adsorption study}

A stock solution of MB $(2000 \mathrm{mg} / \mathrm{L})$ was prepared and diluted to obtain the required experimental concentrations. The batch experiments were performed with $20 \mathrm{~mL}$ of known $\mathrm{MB}$ dye solution in the presence of $0.01 \mathrm{~g}$ of $\mathrm{cl}-\mathrm{CS}-\mathrm{p}(\mathrm{MA}) / \mathrm{GO}-\mathrm{Fe}_{3} \mathrm{O}_{4} \mathrm{NPs}$ as the adsorbent in $50-\mathrm{mL}$ Falcon tubes. The Falcon tubes were placed in an electrically operated multishaking incubator at $25^{\circ} \mathrm{C}$ and 120 rpm. We examined a range of $\mathrm{MB}$ concentrations $(100-1,700 \mathrm{mg} / \mathrm{L})$ for isotherm study at $\mathrm{pH} 8.5 \pm 0.1$. All adsorption studies were performed twice. A kinetic study was performed using $100 \mathrm{mg} / \mathrm{L}$ of $\mathrm{MB}$ dye and cl-CS-p (MA)/GO- $\mathrm{Fe}_{3} \mathrm{O}_{4} \mathrm{NPs}$ at $\mathrm{pH} 8.5 \pm 0.1$. The experimental procedures are described in detail in the Supporting Information. The \% of $\mathrm{MB}$ adsorption and equilibrium uptake capacity were determined using equations (1) and (2), respectively. A detailed explanation behind these calculations is given in the Supporting Information.

$$
\begin{aligned}
& \% \text { Adsorption }=\frac{\left(C_{0}-C_{e}\right)}{C_{0}} \times 100 \\
& \text { Equilibrium uptake capacity }=\left(C_{0}-C_{e}\right) \times \frac{V}{W}
\end{aligned}
$$

\section{Effect of dye type on removal efficiency}

The efficiency of the cl-CS-p(MA)/GO- $\mathrm{Fe}_{3} \mathrm{O}_{4} \mathrm{NPs}$ in removing different types of dyes was examined with two dye mixtures: mixture I $(\mathrm{MB}+\mathrm{TB})$ and mixture II $(\mathrm{MB}+$ $\mathrm{MO})$. Mixture I included two cationic dyes (MB and $\mathrm{TB}$ ) at $10 \mathrm{mg} / \mathrm{L}$ each; mixture II contained cationic $\mathrm{MB}$ and anionic $\mathrm{MO}$ at $10 \mathrm{mg} / \mathrm{L}$ each. To evaluate the adsorption behaviors of the dye mixtures, $0.01 \mathrm{~g}$ of cl-CS-p(MA)/ $\mathrm{GO}-\mathrm{Fe}_{3} \mathrm{O}_{4} \mathrm{NPs}$ was brought into contact with $20 \mathrm{~mL}$ of each dye mixture at $\mathrm{pH} 8.5$ in a shaking incubator at $25^{\circ} \mathrm{C}$ and $120 \mathrm{rpm}$.

\section{Stability study of cl-CS-p(MA)/GO- $\mathrm{Fe}_{3} \mathrm{O}_{4} \mathrm{NPs}$}

The stability of the adsorbents over a wide range of $\mathrm{pH}$ values is a critical parameter toward ensuring that the adsorbents do not disperse or solubilize upon contact with aqueous media. Thus, a fixed amount of cl-CS-p (MA)/GO- $\mathrm{Fe}_{3} \mathrm{O}_{4} \mathrm{NPs}$ was separately added into $20 \mathrm{~mL}$ of acidic ( $\mathrm{pH} 3 \pm 0.5)$, neutral ( $\mathrm{pH} 7 \pm 0.5)$, or basic ( $\mathrm{pH} 9 \pm$ $0.5)$ solution in $50-\mathrm{mL}$ Falcon tubes. The Falcon tubes were placed in the incubator for $24 \mathrm{~h}$ at $25^{\circ} \mathrm{C}$. During that 

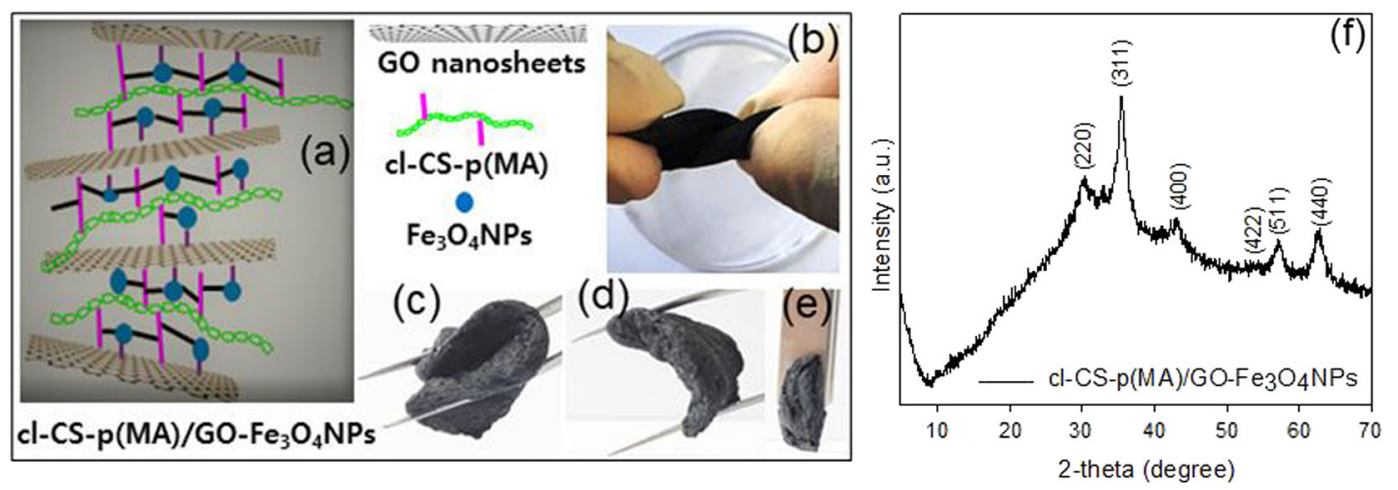

Fig. 1 a Schematic, b-d flexibility test, e magnetic property, and $\mathbf{f} X R D$ pattern of cl-CS-p(MA)/GO-Fe $\mathrm{O}_{4} N \mathrm{NPS}$

time, the structural stability of the cl-CS-p(MA)/ $\mathrm{GO}-\mathrm{Fe}_{3} \mathrm{O}_{4} \mathrm{NPs}$ was monitored.

\section{Recyclability test}

An ideal adsorbent must have regeneration properties. Thus, MB-loaded cl-CS-p(MA)/GO-Fe $\mathrm{O}_{4} \mathrm{NPs}$ were recycled from the experimental aqueous media using an external magnet or spatula based on a desorption process. For the desorption study, $100 \mathrm{mg} / \mathrm{L}$ of $20 \mathrm{~mL}$ of $\mathrm{MB}$ adsorption was carried out in the presence of $0.01 \mathrm{~g}$ of the cl-CS-p(MA)/GO- $\mathrm{Fe}_{3} \mathrm{O}_{4} \mathrm{NPs}$. The separated MB-loaded cl-CS-p(MA)/GO- $\mathrm{Fe}_{3} \mathrm{O}_{4} \mathrm{NP}$ nanocomposite was freezedried. After drying, the MB-loaded cl-CS-p(MA)/ GO- $\mathrm{Fe}_{3} \mathrm{O}_{4} \mathrm{NP}$ nanocomposite was treated with $20 \mathrm{~mL}$ (aqueous) of $0.05 \mathrm{M} \mathrm{HCl}$ solution and placed in the shaking incubator. After a fixed time interval, the supernatant solution was analyzed using a UV-VIS spectrophotometer. The same process was repeated at least five times.

\section{Results and discussion}

This synthesis approach relies on the functionalization of MA onto the backbone of CS in the presence of MBA as the cross-linker. Free radical sites were generated on the CS backbones using KPS as an initiator at $65^{\circ} \mathrm{C}$ and then were allowed to react with $\mathrm{MA}$, which underwent cross-linking polymerization through chain reactions in the presence of the reactive sites of MBA to form a 3D cross-linked polymer hydrogel (Fig. 1a; Scheme S1, Supporting Information). The resulting $\mathrm{cl}-\mathrm{CS}-\mathrm{p}(\mathrm{MA})$ hydrogel was used as a gelling matrix to stabilize the GO layers. Then, the synthesized cl-CS-p(MA)/GO underwent further cross-linking with in situ- developed $\mathrm{Fe}_{3} \mathrm{O}_{4} \mathrm{NPs}$. The presence of $\mathrm{GO}$ in the nanocomposite provided the overall stability of the cross-linked 3Dnanostructured material (Fig. 1a; Scheme S1, Supporting Information). Herein, a stable dispersion of unstacked GO nanosheets was prepared in the presence of the gelling matrix of cl-CS-p(MA). Next, the stable gelling dispersion of the GO nanosheets and carboxylate side chains of cl-CS-p(MA) minimized the possibility of $\mathrm{Fe}_{3} \mathrm{O}_{4} \mathrm{NP}$ agglomeration; this stabilization effect was achieved through cross-linking reactions and led to the development of a sponge-like material with magnetic behavior (Fig. 1(b-e), inset images).

Several characterization techniques were utilized to confirm the development of nanocomposites through polymerization and in situ cross-linking. The XRD spectrum of the cl-CS-p(MA)/GO- $\mathrm{Fe}_{3} \mathrm{O}_{4} \mathrm{NPs}$ shows distinct diffraction peaks at $2 \theta=(30.11,35.39,42.85,53.43,57.07$, and 62.66$)^{\circ}$ which are ascribed to the (220), (311), (400), (422), (511), and (440) crystal planes of the $\mathrm{Fe}_{3} \mathrm{O}_{4} \mathrm{NPs}$, respectively (JCPDS 19-0629) (Fig. 1f) ${ }^{28}$. In the same spectrum, the absence of the (002) crystal plane at $2 \theta=$ $11.25^{\circ}$ for GO (present in the bare GO spectrum; Figure S1, Supporting Information) corresponds to the disrupted GO layers and indicates unstacked graphene sheets $^{29}$. The presence of low-intensity peaks in cl-CS-p (MA) can contribute to the generation of a poorly crystalline material originating from cross-linked chitosan. Additionally, it should be noted that the crystallinity present in CS was decreased in cl-CS-p(MA) due to the effect of the cross-linking and polymerization reactions (Scheme S1, Supporting Information) ${ }^{30}$. Similarly, the Raman spectra (Figure S2, Supporting Information) reveal a continuously increasing intensity ratio $\left(\mathrm{I}_{\mathrm{D}} / \mathrm{I}_{\mathrm{G}}\right)$ of graphite to GO and cl-CS-p(MA)/GO-Fe $\mathrm{O}_{3}$ NPs. These observations indicate that the graphite has oxidized (Figure S2, green spectrum, Supporting Information) and that the cl-CS-p(MA) has been functionalized in the presence of $\mathrm{GO}$ and the in situ- developed $\mathrm{Fe}_{3} \mathrm{O}_{4} \mathrm{NPs}$ (Figure S2, blue spectrum, Supporting Information), thereby inhibiting graphene restacking ${ }^{31}$. The FT-IR spectrum of cl-CS-p(MA) contains all of the characteristic peaks of CS with several additional peaks, revealing the formation of a cross-linked graft copolymer 

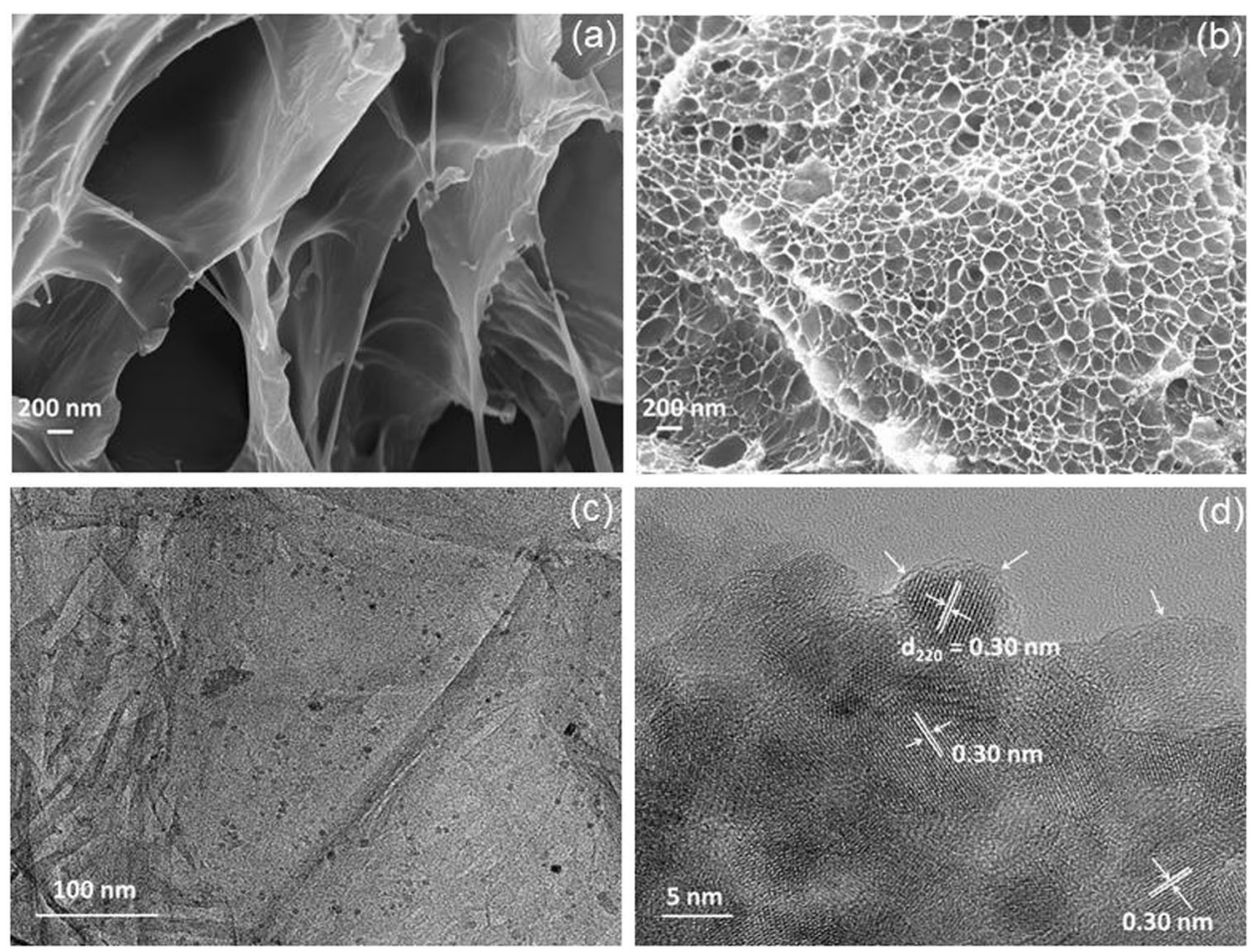

Fig. 2 FE-SEM images of $\mathbf{a} \mathrm{Cl}-\mathrm{CS}-\mathrm{p}(\mathrm{MA})$ and $\mathbf{b} \mathrm{Cl}-\mathrm{CS}-\mathrm{p}(\mathrm{MA}) / \mathrm{GO}-\mathrm{Fe}_{3} \mathrm{O}_{4} \mathrm{NPS}$; and $\mathbf{c} \mathrm{FE}-\mathrm{TEM}$ and $\mathbf{d} \mathrm{HR}-\mathrm{FE}-\mathrm{TEM}$ images of cl-CS-p(MA)/GO-Fe $\mathrm{O}_{4} \mathrm{NPs}$

(Figure S3a, b, Supporting Information). Meanwhile, the corresponding nanocomposite cl-CS-p(MA)/ $\mathrm{GO}-\mathrm{Fe}_{3} \mathrm{O}_{4} \mathrm{NPs}$ shows all of the characteristic peaks of $\mathrm{cl}-\mathrm{CS}-\mathrm{p}(\mathrm{MA})$ and GO, although the peak positions have shifted, indicating the successful formation of the nanocomposite (Figure S3c, d, Supporting Information) ${ }^{32}$. FESEM analysis reveals that the fibrillar morphology of CS changes to a completely porous morphology in cl-CS-p (MA) (Fig. 2a; Figure S4a, Supporting Information). The surface morphology of the cl-CS-p(MA)/GO-Fe $\mathrm{O}_{4} \mathrm{NPs}$ was completely altered to an interconnected microporous network, in contrast to the original microstructures of cl-CS-p(MA) and GO (Fig. 2b; and Figure S4b, Supporting Information). Based on these imaging studies, no surface-deposited $\mathrm{Fe}_{3} \mathrm{O}_{4} \mathrm{NPs}$ were observed, revealing that the in situ-formed $\mathrm{Fe}_{3} \mathrm{O}_{4} \mathrm{NPs}$ were stabilized by the highly dispersed polymeric gelling matrix of the GO nanosheets and were successfully embedded into the $3 \mathrm{D}$ polymeric matrix of cl-CS-p(MA). The EDS spectrum of the $\mathrm{cl}-\mathrm{CS}-\mathrm{p}(\mathrm{MA}) / \mathrm{GO}-\mathrm{Fe}_{3} \mathrm{O}_{4} \mathrm{NPs}$ shows the presence of $\mathrm{Fe}$ in the nanocomposite material (Figure S5a-c, Supporting Information). Similarly, the uniform intensity of an elemental map of Fe suggests that the $\mathrm{Fe}_{3} \mathrm{O}_{4} \mathrm{NPs}$ were properly distributed within the polymeric gelling matrix of GO (Figure S5d, Supporting Information). The FETEM image of the cl-CS-p(MA)/GO-Fe $\mathrm{O}_{4} \mathrm{NPs}$ (Fig. 2c) demonstrates a clearly recognizable, randomly oriented, wrinkled sheet-like structure with high-contrast spherically shaped particles that can be identified as $\mathrm{Fe}_{3} \mathrm{O}_{4} \mathrm{NPs}$. In the HR-FE-TEM image, the GO layer is arranged on the exterior surface of the nanocomposite (indicated by arrows) with the $\mathrm{Fe}_{3} \mathrm{O}_{4} \mathrm{NPs}$ contained in the interior of the material (Fig. 2d). Distinct lattice fringes with an interplanar distance of $0.30 \mathrm{~nm}$ were observed and are consistent with the (220) plane of metallic $\mathrm{Fe}_{3} \mathrm{O}_{4}$ (Fig. 2d) ${ }^{33}$. The high-resolution $\mathrm{C} 1 \mathrm{~s}$ and $\mathrm{N} 1 \mathrm{~s}$ XPS spectra of CS, cl-CS-p(MA), cl-CS-p(MA)/ $\mathrm{GO}-\mathrm{Fe}_{3} \mathrm{O}_{4} \mathrm{NPs}$, and $\mathrm{GO}$ and the $\mathrm{Fe} 2 \mathrm{p}$ spectrum of cl-CS-p(MA)/GO-Fe $\mathrm{O}_{4} \mathrm{NPs}$ are compared in Fig. 3 and Figure S6, Supporting Information ${ }^{34-36}$. The $C 1$ s spectrum of the cl-CS-p(MA) contains three components associated with $\mathrm{CS}$, corresponding to different carbon functionalities at approximately $284.6 \mathrm{eV}(\mathrm{C}-\mathrm{C} / \mathrm{C}-\mathrm{H})$, $285.5 \mathrm{eV}$ ( $\mathrm{C}-\mathrm{N}$ from glucosamine ring and cross-linker), and $288.8 \mathrm{eV}(\mathrm{O}-\mathrm{C}=\mathrm{O} / \mathrm{N}-\mathrm{C}=\mathrm{O})$ with the absence of a peak at $286.45 \mathrm{eV}$ (in chitosan), indicating that the $\mathrm{C}-\mathrm{OH}$ group was effectively grafted and cross-linked (Fig. 3a, b). The $\mathrm{C} 1 \mathrm{~s}$ spectrum of the $\mathrm{cl}-\mathrm{CS}-\mathrm{p}(\mathrm{MA}) / \mathrm{GO}-\mathrm{Fe}_{3} \mathrm{O}_{4} \mathrm{NP}$ nanocomposite demonstrates similar effects as those observed for cl-CS-p(MA) with regard to the characteristic peaks of these materials (Fig. 3c). However, the altered peak intensity of $\mathrm{C}-\mathrm{C} / \mathrm{C}-\mathrm{H}$ was observed in the spectrum of $\mathrm{cl}-\mathrm{CS}-\mathrm{p}(\mathrm{MA}) / \mathrm{GO}-\mathrm{Fe}_{3} \mathrm{O}_{4} \mathrm{NPs}$ relative to those of $\mathrm{cl}-\mathrm{CS}-\mathrm{p}(\mathrm{MA})$ and $\mathrm{GO}$ (Fig. 3c; Figure S6a, 

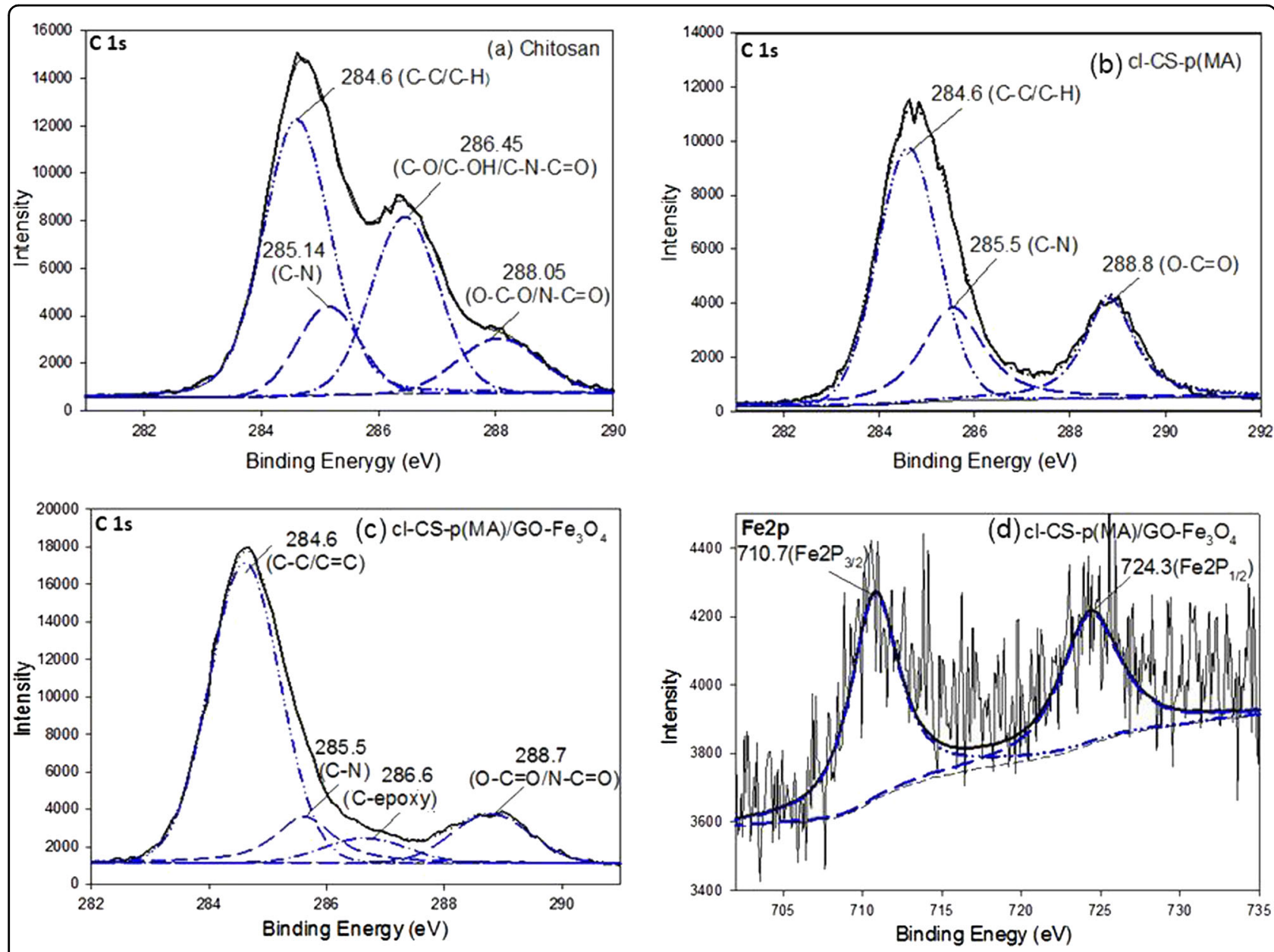

Fig. 3 High-resolution XPS spectra (C 1s) with deconvoluted peaks of a chitosan, $\mathbf{b}$ cl-CS-p(MA), c cl-CS-p(MA)/GO-Fe $\mathrm{O}_{4} \mathrm{NPs}$, and $\mathbf{d}$ Fe $2 \mathrm{p}$ spectrum of $\mathrm{cl}-\mathrm{CS}-\mathrm{p}(\mathrm{MA}) / \mathrm{GO}-\mathrm{Fe}_{3} \mathrm{O}_{4} \mathrm{NPs}$

Supporting Information), suggesting that the nanocomposite was successfully fabricated with a good interaction between the phases. Additionally, the decrease in the peak intensity of the c-(epoxy group) in the cl-CS-p (MA)/GO- $\mathrm{Fe}_{3} \mathrm{O}_{4} \mathrm{NPs}$ compared to GO reveals that most of the epoxy groups in $\mathrm{GO}$ undergo functionalization with cl-CS-p(MA) (Figure S6a, Supporting Information). The Fe 2p spectrum (Fig. 3d) reveals the characteristic peaks of $\mathrm{Fe}_{3} \mathrm{O}_{4} \mathrm{NPs}$ at $710.7 \mathrm{eV}\left(\mathrm{Fe} \mathrm{2} \mathrm{p}_{3 / 2}\right)$ and $724.3 \mathrm{eV}\left(\mathrm{Fe} \mathrm{2} \mathrm{p}_{1 / 2}\right)^{37}$, signifying the successful embedding of $\mathrm{Fe}_{3} \mathrm{O}_{4} \mathrm{NPs}$ within the polymeric gelling GO matrix. The decrease in intensity of the $\mathrm{N} 1 \mathrm{~s}$ spectrum of $\mathrm{C}-\mathrm{N}$ for $\mathrm{cl}-\mathrm{CS}-\mathrm{p}(\mathrm{MA})$ is more obvious than the $\mathrm{C}-\mathrm{N}$ spectrum for CS (Figure S6b, c, Supporting Information), suggesting that some of the $\mathrm{C}-\mathrm{N}$ groups can participate in the free radical polymerization reaction. The $\mathrm{N} 1 \mathrm{~s}$ spectrum of $\mathrm{cl}-\mathrm{CS}-\mathrm{p}(\mathrm{MA})$ at $401.5 \mathrm{eV}^{38}$ (Figure S6c Supporting Information) suggests the presence of unreacted, positively charged ammonium groups, which are responsible for the stable gelling dispersion of GO. Furthermore, the higher binding energies of $\mathrm{N} 1 \mathrm{~s}$ in cl-CS$-\mathrm{p}(\mathrm{MA}) / \mathrm{GO}-\mathrm{Fe}_{3} \mathrm{O}_{4} \mathrm{NPs}$ relative to those recorded for the $\mathrm{N} 1 \mathrm{~s}$ spectra of $\mathrm{cl}-\mathrm{CS}-\mathrm{p}(\mathrm{MA})$ and GO indicate the successful formation of the nanocomposite (Figure S6c-e Supporting Information) ${ }^{39}$.

The aforementioned cl-CS-p(MA)/GO- $\mathrm{Fe}_{3} \mathrm{O}_{4} \mathrm{NP}$ nanocomposite was used for preliminary adsorption studies. The adsorption efficiency increased with the solution $\mathrm{pH}$ and reached at almost constant value. (Figure S7, Supporting Information). Based on this $\mathrm{pH}$ effect, $\mathrm{pH} 8.5$ was chosen for additional experiments. To understand the surface behavior of the nanocomposite material in aqueous solutions, the point of zero charge $\left(\mathrm{pH}_{\mathrm{pzc}}\right)$ was measured by the $\mathrm{pH}$ drift method. As shown in Figure S8 (Supporting Information), the $\mathrm{pH}_{\mathrm{pzc}}$ of the cl-CS-p(MA)/GO-Fe $\mathrm{O}_{4} \mathrm{NPs}$ was 6.53 , indicating that the nanocomposite bears negative surface charges at solution $\mathrm{pH}>6.53$. Therefore, at $\mathrm{pH} 8.5$, the electrostatic interaction between the cationic $\mathrm{MB}$ $\left(\mathrm{C}_{16} \mathrm{H}_{18} \mathrm{~N}_{3} \mathrm{~S}^{+}\right)^{12}$ and the anionic cl-CS-p(MA)/ $\mathrm{GO}-\mathrm{Fe}_{3} \mathrm{O}_{4} \mathrm{NPs}$ can predominate and is responsible for the high adsorption efficiency of the material. 
The adsorption isotherm is critical for interpreting the interactive behavior between the nanocomposite and the MB molecules ${ }^{40}$. The elucidated equilibrium data were fitted using the nonlinear Langmuir model ${ }^{41}$, Freundlich model $^{42}$, and Sips model ${ }^{43}$. The estimated parameters are presented in Table 1 with their associated correlation coefficients. The Langmuir model fits the experimental data better than the Freundlich model. The Sips model, which is a combination of the Langmuir and Freundlich models, was found to be the best-fit model based on the high correlation coefficient of $R^{2}=0.9918$. At high adsorbate concentrations, the Sips model follows the monolayer adsorption behavior that is characteristic of the Langmuir model ${ }^{44}$. The maximum uptake capacity of $2478 \mathrm{mg} / \mathrm{g}$ for MB dye is a significant result with regard to reports for other materials (Fig. $4 \mathrm{a}$ and Table S1, Supporting Information $)^{7,17-19,22,23,45-49}$.

Table 1 Isotherm model parameters for MB adsorption using $\mathrm{cl}-\mathrm{CS}-\mathrm{p}(\mathrm{MA}) / \mathrm{GO}-\mathrm{Fe}_{3} \mathrm{O}_{4} \mathrm{NPs}$ as the adsorbent

\begin{tabular}{lll}
\hline Isotherm model & Parameter & Results \\
\hline Langmuir & $\mathrm{q}_{\max }(\mathrm{mg} / \mathrm{g})$ & 2478 \\
& $\mathrm{~b}(\mathrm{~L} / \mathrm{mg})$ & 0.366 \\
& $R^{2}$ & 0.9668 \\
Freundlich & $\mathrm{K}_{\mathrm{F}}(\mathrm{L} / \mathrm{mg})^{1 / \mathrm{n}}$ & 1084 \\
& $\mathrm{n}$ & 7.182 \\
& $R^{2}$ & 0.7999 \\
Sips & $\mathrm{q}_{\mathrm{m}}(\mathrm{mg} / \mathrm{g})$ & 2409 \\
& $\mathrm{~K}_{\mathrm{s}}$ & 0.369 \\
& $\mathrm{~m}_{\mathrm{s}}$ & 1.783 \\
& $R^{2}$ & 0.9918 \\
\hline
\end{tabular}

The kinetic efficiency of the adsorbent material allows for the rapid decontamination of toxic organic dyes and offers a prospect for use in the area of real applications. The nanocomposite can easily remove $~ 99 \% \mathrm{MB}$ dye at equilibrium, which was reached within $20 \mathrm{~min}$ of exposure in our studies. After prolonging the contact time to 1 $\mathrm{h}$, the water was completely MB-free (Figure S9). The obtained kinetic data were suitably fit with a nonlinear pseudo-first-order kinetic model $\left(R^{2}=0.9944\right)$ and pseudo-second-order kinetic model $\left(R^{2}=0.9836\right)$ (Fig. 4b, Table S2; Supporting Information $)^{50,51}$. The extraordinary uptake capacity and rapid removal of $\mathrm{MB}$ are attributed to the synergistic development of the functionalized nanostructured material. To explain this phenomenon, we conducted several adsorption studies by controlling the synthesized products (Fig. 5a). Specifically, pure crosslinked copolymer cl-CS-p(MA) and cl-CS-p(MA)/GO showed low $M B$ dye adsorption performance (Fig. 5a). Meanwhile, the removal of $\mathrm{MB}$ by $\mathrm{cl}-\mathrm{CS}-\mathrm{p}(\mathrm{MA}) /$ $\mathrm{Fe}_{3} \mathrm{O}_{4} \mathrm{NPs}$ was comparable to that of cl-CS-p(MA)/ $\mathrm{GO}-\mathrm{Fe}_{3} \mathrm{O}_{4} \mathrm{NPs}$, but the recyclability of cl-CS-p(MA)/ $\mathrm{Fe}_{3} \mathrm{O}_{4} \mathrm{NPs}$ was significantly lower (Fig. 5a). This observation indicates that the role of $\mathrm{GO}$ is crucial in the fabrication of a stable and recyclable nanocomposite material. In fact, the sequential syntheses of the crosslinked chitosan and stabilized GO nanosheets and further cross-linking with the $\mathrm{Fe}_{3} \mathrm{O}_{4} \mathrm{NPs}$ constitute a deliberate approach toward producing a structurally stable material with many well-dispersed binding sites that can deliver faster adsorption behavior with higher capacities. It is important to note that the main binding sites for $\mathrm{MB}$ adsorption are the carboxylate chains of poly(MA). More insight into the performance of $\mathrm{cl}-\mathrm{CS}-\mathrm{p}(\mathrm{MA}) /$ $\mathrm{GO}-\mathrm{Fe}_{3} \mathrm{O}_{4} \mathrm{NPs}$ toward $\mathrm{MB}$ removal can be gleaned by using a combination of XPS, FE-SEM, and elemental mapping analyses. The inclusion of $\mathrm{MB}$ within the
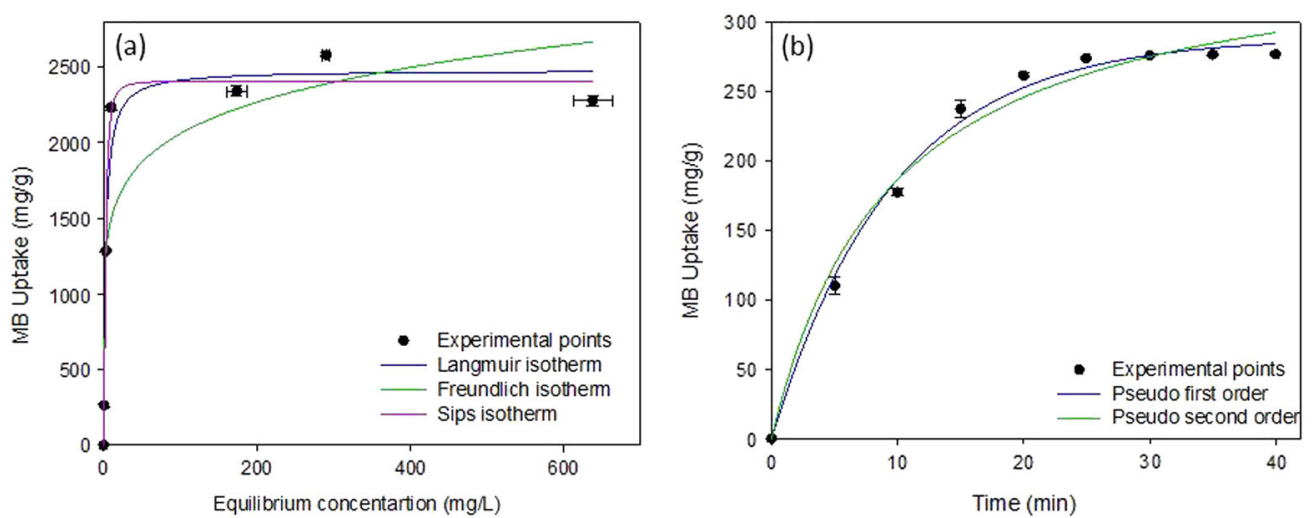

Fig. 4 a Nonlinear fitting of adsorption isotherms and $\mathbf{b}$ nonlinear fitting of kinetic models for the adsorption of MB dye on $\mathrm{Cl}-\mathrm{CS}-\mathrm{p}(\mathrm{MA}) /$ $\mathrm{GO}-\mathrm{Fe}_{3} \mathrm{O}_{4} \mathrm{NPS}$ 

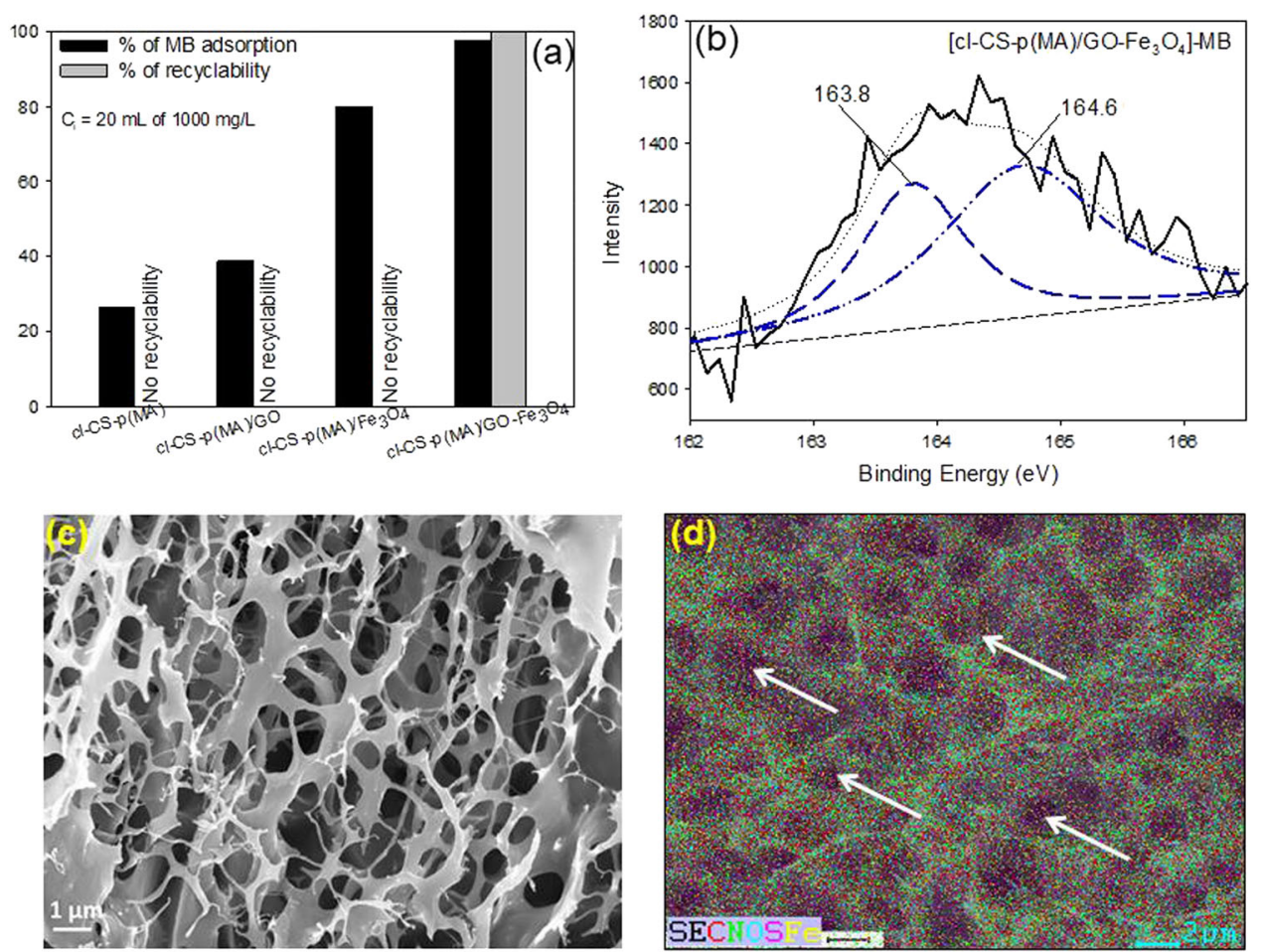

Fig. 5 a Comparative \% of MB adsorption and recyclability using different adsorbents. b High-resolution XPS spectrum (S 2p) with deconvoluted peaks of MB-adsorbed cl-CS-p(MA)/GO-Fe $\mathrm{O}_{4} \mathrm{NPS}$, c FE-SEM image and $\mathbf{d}$ elemental map of MB-adsorbed cl-CS-p(MA)/GO-Fe $\mathrm{O}_{4} \mathrm{NPs}$ (the arrows indicate the presence of concentrated sulfur in the pores)

cl-CS-p(MA)/GO- $\mathrm{Fe}_{3} \mathrm{O}_{4} \mathrm{NPs}$ was revealed based on the appearance of $\mathrm{S} 2 \mathrm{p}$ peaks at 163.8 and $164.6 \mathrm{eV}$ for $-\mathrm{C}-\mathrm{S}-\mathrm{C} /-\mathrm{C}=\mathrm{S}-$, respectively (Fig. $5 \mathrm{~b}$ ). Figure S10a (Supporting Information) shows the characteristics of the $\mathrm{S} 2 \mathrm{p}$ peaks $^{52}$ for $\mathrm{MB}$. The strong binding interaction between the sulfur atom and carboxylate group present in $\mathrm{cl}-\mathrm{CS}-\mathrm{p}(\mathrm{MA}) / \mathrm{GO}-\mathrm{Fe}_{3} \mathrm{O}_{4} \mathrm{NPs}$ was verified based on the changes in intensity and the shifts of the $\mathrm{C} 1 \mathrm{~s}$ peaks in the MB-adsorbed cl-CS-p(MA)/GO- $\mathrm{Fe}_{3} \mathrm{O}_{4} \mathrm{NPs}$ (Figure S9b, Supporting Information). The FE-SEM micrographs of the MB-adsorbed cl-CS-p(MA)/GO-Fe ${ }_{3} \mathrm{O}_{4} \mathrm{NPs}$ (Fig. 5c; Figure S11, Supporting Information) showed micropores with interconnected channels. The specific surface area of the nanocomposite was very small $\left(1.91 \mathrm{~m}^{2} / \mathrm{g}\right)$ compared with that of porous adsorbents, i.e., activated carbons, which are known to exhibit values up to $\sim 3000 \mathrm{~m}^{2} / \mathrm{g}$. The mean pore diameter of the nanocomposite was $21.83 \mathrm{~nm}$. Here, it should be noted that the developed nanocomposite possesses a structure that is similar to that of a hydrogel, in which the uniform distribution of active binding sites throughout the hydrogel is crucial for obtaining the observed outstanding adsorption performance, in contrast to a high surface area and/or porosity. The micropores of the nanocomposite are not directly responsible for the adsorption property but instead serve as microchannels for easy penetration of the MB dye.
Herein, the development of interconnected microchannels imparts a shorter diffusion path, enhancing the kinetics of $\mathrm{MB}$ uptake. Additionally, the elemental map of sulfur in the MB-adsorbed cl-CS-p(MA)/GO- $\mathrm{Fe}_{3} \mathrm{O}_{4} \mathrm{NPs}$ displayed the distribution of sulfur on the surface of the nanocomposite, signifying the uptake of MB (Fig. 5d). The surface adsorption of $\mathrm{MB}$ dye on the cl-CS-p(MA)/ $\mathrm{GO}-\mathrm{Fe}_{3} \mathrm{O}_{4} \mathrm{NP}$ nanocomposite was further confirmed by the presence of sulfur in the EDS spectra of MB-adsorbed cl-CS-p(MA)/GO-Fe ${ }_{3} \mathrm{O}_{4} \mathrm{NPs}$ (Figure S12, Supporting Information). It is important to note that the microchannels were active throughout the entire contact time and that even after the fifth adsorption-desorption cycle, the pores were capable of uptaking the cationic MB dye. Furthermore, the separation of the adsorbed dye from (i.e., the regeneration of) the MB-adsorbed swollen cl-CS-p(MA)/GO- $\mathrm{Fe}_{3} \mathrm{O}_{4} \mathrm{NPs}$ was achieved with a simple approach, namely, by changing the $\mathrm{pH}$ of the aqueous media. Moreover, the substrate could be regenerated at least five times through an adsorption-desorption cycle by using an external magnet or spatula, achieving an adsorption efficiency that was greater than $~ 97 \%$ (Figure S13, Supporting Information). The magnetic hysteresis of the cl-CS-p(MA)/GO- $\mathrm{Fe}_{3} \mathrm{O}_{4} \mathrm{NPs}$ was measured, and the saturation magnetization was found to be 6.1 $\mathrm{emu} / \mathrm{g}$, demonstrating magnetic separation ability 
(Figure S14, Supporting Information). After obtaining such promising results in the removal of MB from synthetic wastewater, we checked the stability of the adsorbate material (cl-CS-p(MA)/GO-Fe $\mathrm{O}_{4} \mathrm{NPs}$ ) under different experimental conditions such as $\mathrm{pH} 9 \pm 0.5, \mathrm{pH}$ $7 \pm 0.5$, and $\mathrm{pH} 3 \pm 0.5$ at $25^{\circ} \mathrm{C}$ for $24 \mathrm{~h}$ to investigate its suitability in practical applications. The nanostructured material swelled but did not disperse under these experimental conditions, revealing that it possesses both chemical and mechanical stability in the ranges tested (Table S3; Figure S15, Supporting Information).

The adsorption behavior of the cl-CS-p(MA)/ GO- $\mathrm{Fe}_{3} \mathrm{O}_{4} \mathrm{NPs}$ in several dye mixtures was examined. The UV-Vis absorption spectrum of the cationic dye mixture $(\mathrm{MB}+\mathrm{TB})$ is shown in Figure S16 (Supporting Information). After adsorption, both $\mathrm{MB}$ and $\mathrm{TB}$ were completely removed by the cl-CS-p(MA)/GO- $\mathrm{Fe}_{3} \mathrm{O}_{4} \mathrm{NPs}$ within 10 min. Based on this observation, it can be noted that the developed nanocomposite does not exhibit adsorbent behavior that is specific to $\mathrm{MB}$ but can adsorb any positively charged dye. From the practical application point of view, this would be a favorable characteristic of the nanocomposite because it suggests that all types of cationic dyes could be simultaneously removed from wastewater using the developed nanocomposite. In another experiment, we tested the adsorption behavior of the nanocomposite in a mixed-species scenario with an anionic dye, MO, in addition to the cationic dye MB (Figure S17, Supporting Information). Following this study, the characteristic peak of $\mathrm{MB}$ was observed to almost completely disappear, whereas the removal of the anionic MO was not significant ( 11\%). The inset photograph in Figure S17 shows that the color was due to $\mathrm{MO}$ remaining after adsorption and magnetic separation, supporting again our understanding that the main binding mechanism of cl-CS-p(MA)/ $\mathrm{GO}-\mathrm{Fe}_{3} \mathrm{O}_{4} \mathrm{NPs}$ is via electrostatic interaction. Therefore, to clean up wastewater containing both cationic and anionic dyes, an anionic adsorbent should be used in conjunction with the developed cationic adsorbent.

\section{Conclusion}

The studies presented here revealed a successful synthesis strategy for the development of a functionalized magnetic nanocomposite adsorbent, cl-CS-p(MA)/ $\mathrm{GO}-\mathrm{Fe}_{3} \mathrm{O}_{4} \mathrm{NPs}$, with outstanding adsorption performance with dyes. The cl-CS-p(MA)/GO-Fe $\mathrm{O}_{4} \mathrm{NP}$ nanocomposite demonstrated a maximum adsorption capacity of $2478 \mathrm{mg} / \mathrm{g}$, which is the highest reported performance so far. The efficient removal ability of this nanocomposite was likely because of the formation of well-dispersed active binding sites and interconnected microchannels, both of which facilitated rapid adsorption. Furthermore, the cl-CS-p(MA)/GO- $\mathrm{Fe}_{3} \mathrm{O}_{4} \mathrm{NP}$ nanocomposite was stable over a wide range of $\mathrm{pH}$ values, and after adsorption, the cl-CS-p(MA)/GO- $\mathrm{Fe}_{3} \mathrm{O}_{4} \mathrm{NPs}$ could be separated using a magnet or spatula. These outstanding properties make cl-CS-p(MA)/GO-Fe $\mathrm{O}_{4} \mathrm{NPs}$ an attractive candidate material in the field of water environmental remediation.

\section{Acknowledgements}

This work was supported by the Korean Government through NRF (2017R1A2A1A05001207) grants. A.K.S. gratefully acknowledges the BK 21 Plus postdoctoral fellowship for financial assistance. The authors are thankful to the CURF and KBSI, Jeonju center for instrumental analyses.

\section{Conflict of interest}

The authors declare that they have no conflict of interest.

\section{Publisher's note}

Springer Nature remains neutral with regard to jurisdictional claims in published maps and institutional affiliations.

Supplementary information is available for this paper at https://doi.org/ 10.1038/s41427-018-0104-8.

Received: 8 June 2018 Revised: 24 October 2018 Accepted: 5 November 2018.

Published online: 23 January 2019

\section{References}

1. Khin, M. M., Nair, A. S., Babu, V. J., Murugan, R. \& Ramakrishna, S. A review on nanomaterials for environmental remediation. Energy \& Environ. Sci. 5, 8075-8109 (2012)

2. Cheng, H. N., Doemeny, L. J., Geraci, C. L. \& Grob Schmidt, D. Nanotechnology Overview: Opportunities and Challenges in Nanotechnology: Delivering on the Promise Vol 1, (eds. Cheng, H. N., Doemeny, L. J., Geraci, C. L. \& Grob Schmidt, D.) Ch. 1, 1-12 (American Chemical Society, USA, 2016).

3. Nardecchia, S., Carriazo, D., Ferrer, M. L., Gutierrez, M. C. \& del Monte, F. Three dimensional macroporous architectures and aerogels built of carbon nanotubes and/or graphene: synthesis and applications. Chem. Soc. Rev. 42, 794-830 (2013).

4. Dongyun, $C$. et al. Micro-nanocomposites in environmental management. Adv. Mater. 28, 10443-10458 (2016).

5. Byun, J., Patel, H. A., Thirion, D. \& Yavuz, C. T. Charge-specific size-dependent separation of water-soluble organic molecules by fluorinated nanoporous networks. Nat. Commun. 7, 13377 (2016).

6. Aguila, B. et al. Efficient mercury capture using functionalized porous organic polymer. Advanced Materials 29, 1-6 (2017).

7. Hameed, B. H., Din, A. T. M. \& Ahmad, A. L. Adsorption of methylene blue onto bamboo-based activated carbon: Kinetics and equilibrium studies. J. Hazard. Mater. 141, 819-825 (2007).

8. Wang, S. \& Peng, Y. Natural zeolites as effective adsorbents in water and wastewater treatment. Chem. Eng. J. 156, 11-24 (2010)

9. Peng, N. et al. Superabsorbentcellulose-clay nanocomposite hydrogels for highly efficient removal of dye in water. ACS Sustain. Chem. \& Eng. 4, 7217-7224 (2016)

10. Sarkar, A. K., Saha, A., Panda, A. B. \& Pal, S. pH Triggered superior selective adsorption and separation of both cationic and anionic dyes and photocatalytic activity on a fully exfoliated titanate layer-natural polymer based nanocomposite. Chem. Commun. 51, 16057-16060 (2015).

11. Zhuang, X., Wan, Y., Feng, C., Shen, Y. \& Zhao, D. Highly efficient adsorption of bulky dye molecules in wastewater on ordered mesoporous carbons. Chem. Mater. 21, 706-716 (2009).

12. Homaeigohar, S., Zillohu, A., Abdelaziz, R., Hedayati, M. \& Elbahri, M. A novel nanohybrid nanofibrous adsorbent for water purification from dye pollutants. Materials 9, 848 (2016).

13. Shahin, H., Thomas, S., Julian, S., Lorenz, K. \& Mady, E. A flexible oxygenated carbographite nanofilamentous buckypaper as an amphiphilic membrane. Adv. Mater. Interfaces 5, 1800001 (2018). 
14. Janaki, V. et al. Polyaniline/chitosan composite: an eco-friendly polymer for enhanced removal of dyes from aqueous solution. Synth. Met. 162, 974-980 (2012).

15. Compton, O. C. \& Nguyen, S. T. Graphene oxide, highly reduced graphene oxide, and graphene: versatile building blocks for carbon-based materials. Small 6, 711-723 (2010).

16. Yan, $\mathrm{H}$. et al. Influence of the surface structure of graphene oxide on the adsorption of aromatic organic compounds from water. ACS Appl. Mater. \& Interfaces 7, 6690-6697 (2015).

17. Liu, F., Chung, S., Oh, G. \& Seo, T. S. Three-dimensional graphene oxide nanostructure for fast and efficient water-soluble dye removal. ACS Appl. Mater. \& Interfaces 4, 922-927 (2012).

18. Zhang, J. et al. Poly(acrylic acid) functionalized magnetic graphene oxide nanocomposite for removal of methylene blue. RSC Adv. 5, 32272-32282 (2015).

19. Cheng, Z. et al. One-step fabrication of graphene oxide enhanced magnetic composite gel for highly efficient dye adsorption and catalysis. ACS Sustain. Chem. \& Eng. 3, 1677-1685 (2015).

20. $\mathrm{Bi}, \mathrm{H}$. et al. Highly enhanced performance of spongy graphene as an oil sorbent. J. Mater. Chem. A 2, 1652-1656 (2014).

21. Bao, C. et al. Graphene oxide beads for fast clean-up of hazardous chemicals. J. Mater. Chem. A 4, 9437-9446 (2016).

22. Yang, $Y$. et al. Preparation of reduced graphene oxide/poly(acrylamide) nanocomposite and Its Adsorption of $\mathrm{Pb}(\mathrm{II})$ and methylene blue. Langmuir $\mathbf{2 9}$ 10727-10736 (2013).

23. Yu, R. et al. Graphene oxide/chitosan aerogel microspheres with honeycombcobweb and radially oriented microchannel structures for broad-spectrum and rapid adsorption of water contaminants. ACS Appl. Mater. \& Interfaces $\mathbf{9}$ 21809-21819 (2017).

24. Morrow, B. H., Payne, G. F. \& Shen, J. pH-Responsive self-assembly of polysaccharide through a rugged energy landscape. J. Am. Chem. Soc. 137, 13024-13030 (2015)

25. Hummers, W. S. \& Offeman, R. E. Preparation of graphitic oxide. J. Am. Chem. Soc. 80, 1339-1339 (1958).

26. Marcano, D. C. et al. Improved synthesis of graphene oxide. ACS Nano 4, 4806-4814 (2010).

27. Liu, Q., Zhong, L.-B., Zhao, Q.-B., Frear, C. \& Zheng, Y.-M. Synthesis of Fe3O4/ Polyacrylonitrile Composite electrospun nanofiber mat for effective adsorption of tetracycline. ACS Appl. Mater. \& Interfaces 7, 14573-14583 (2015).

28. Xu, X. et al. Self-sensing, ultralight, and conductive 3D graphene/iron oxide aerogel elastomer deformable in a magnetic field. ACS Nano 9, 3969-3977 (2015).

29. Mondal, A., Maiti, S., Singha, K., Mahanty, S. \& Panda, A. B. TiO2-rGO nanocomposite hollow spheres: large scale synthesis and application as an efficient anode material for lithium-ion batteries. J. Mater. Chem. A 5, 23853-23862 (2017).

30. Yang, X., Tu, Y., Li, L. \& Shang, S. \& Tao X.m. Well-dispersed chitosan/graphene oxide nanocomposites. ACS Appl. Mater. \& Interfaces 2, 1707-1713 (2010).

31. Zhan, Y. et al. Cross-linkable nitrile functionalized graphene oxide/poly(arylene ether nitrile) nanocomposite films with high mechanical strength and thermal stability. J. Mater. Chem. 22, 5602-5608 (2012).

32. Darder, M., Colilla, M. \& Ruiz-Hitzky, E. Biopolymer-clay nanocomposites based on chitosan intercalated in montmorillonite. Chem. Mater. 15, 3774-3780 (2003).
33. Woo, $\mathrm{H}$. et al. Facile synthesis of hybrid $\mathrm{Cu} 2 \mathrm{O} / \mathrm{Pd}-\mathrm{Fe} 3 \mathrm{O} 4$ nanocatalysts for $\mathrm{C}-\mathrm{H}$ arylation of 4-nitroimidazoles. RSC Adv. 6, 36211-36217 (2016).

34. Lopez-Perez, P. M., Marques, A. P., Silva, R. M. Pd, Pashkuleva, I. \& Reis, R. L. Effect of chitosan membrane surface modification via plasma induced polymerization on the adhesion of osteoblast-like cells. J. Mater. Chem. 17, 4064-4071 (2007).

35. Fujii, T. et al. In situ. Phys. Rev. B 59, 3195-3202 (1999).

36. Bo, Z. et al. Green preparation of reduced graphene oxide for sensing and energy storage applications. Sci. Rep. 4, 4684 (2014).

37. Chang, Y. et al. Synthesis of $3 \mathrm{D}$ nitrogen-doped graphene/Fe3O4 by a metal ion induced self-assembly process for high-performance Li-ion batteries. J. Mater. Chem. A 1, 14658-14665 (2013).

38. Le, X. T., Doan, N. D., Dequivre, T., Viel, P. \& Palacin, S. Covalent grafting of chitosan onto stainless steel through aryldiazonium self-adhesive layers. ACS Appl. Mater. \& Interfaces 6, 9085-9092 (2014).

39. Lu, Y.-F. et al. Nitrogen-doped graphene sheets grown by chemical vapor deposition: synthesis and influence of nitrogen impurities on carrier transport. ACS Nano 7, 6522-6532 (2013).

40. Sarkar, A. K., Pal, A., Ghorai, S., Mandre, N. R. \& Pal, S. Efficient removal of malachite green dye using biodegradable graft copolymer derived from amylopectin and poly(acrylic acid). Carbohydr. Polym. 111, 108-115 (2014).

41. Langmuir, I. The adsorption of gases on plane surfaces of glass, mica and platinum. J. Am. Chem. Soc. 40, 1361-1403 (1918).

42. Freundlich, H. M. F. Over the adsorption in solution. J. Phys. Chem. 57, 385-471 (1906).

43. Sips, R. On the structure of a catalyst surface. J. Chem. Phys. 16, 490-495 (1948).

44. Vargas, A. M. M., Cazetta, A. L., Kunita, M. H., Silva, T. L. \& Almeida, V. C. Adsorption of methylene blue on activated carbon produced from flamboyant pods (Delonix regia): Study of adsorption isotherms and kinetic models. Chem. Eng. J. 168, 722-730 (2011).

45. Dolatkhah, A. \& Wilson, L. D. Magnetite/polymer brush nanocomposites with switchable uptake behavior toward methylene blue. ACS Appl. Mater. \& Interfaces 8, 5595-5607 (2016).

46. Hu, L. et al. Fabrication of magnetic water-soluble hyperbranched polyol functionalized graphene oxide for high-efficiency water remediation. Sci. Rep. 6, 28924 (2016).

47. Haque, E., Lo, V., Minett, A. I., Harris, A. T. \& Church, T. L. Dichotomous adsorption behaviour of dyes on an amino-functionalised metal-organic framework, amino-MIL-101(Al). J. Mater. Chem. A 2, 193-203 (2014).

48. Huang, L., He, M., Chen, B., Cheng, Q. \& Hu, B. Facile green synthesis of magnetic porous organic polymers for rapid removal and separation of methylene blue. ACS Sustain. Chem. \& Eng. 5, 4050-4055 (2017).

49. Chen, D., Wang, L., Ma, Y. \& Yang, W. Super-adsorbent material based on functional polymer particles with a multilevel porous structure. Npg Asia Mater. 8, e301 (2016).

50. Lagergren, S. About the theory of so-called adsorption of soluble substances. Handlingar 24, 1-39 (1898).

51. Ho, Y.-S. Second-order kinetic model for the sorption of cadmium onto tree fern: A comparison of linear and non-linear methods. Water Res. 40, 119-125 (2006).

52. Ito, Y., Cong, W., Fujita, T., Tang, Z. \& Chen, M. High catalytic activity of nitrogen and sulfur Co-doped nanoporous graphene in the hydrogen evolution reaction. Angew. Chem. Int. Ed. 54, 2131-2136 (2015). 